
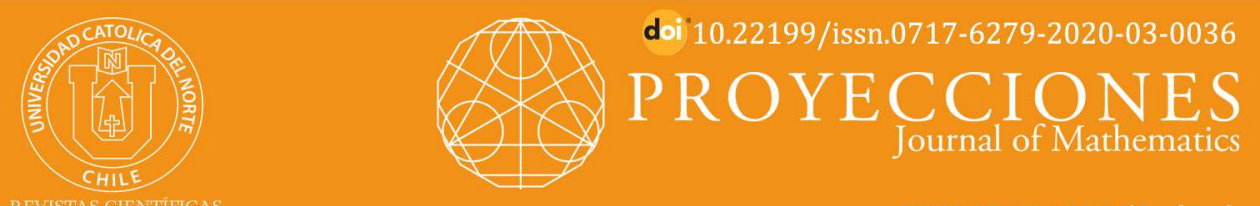

\title{
Maps preserving the square zero of $\eta$-Lie product of operators
}

Ali Taghavi ${ }^{1}$ O orcid.org/0000-0001-6230-733X

Roja Hosseinzadeh² orcid.org/0000-0003-2413-1892

Masoomeh Yousefi ${ }^{3}$

University of Mazandaran, Dept. of Mathematics, Faculty of Mathematical Sciences, Babolsar, Iran.

1曰 taghavi@umzacir; ; ${ }^{2}$ ro.hosseinzadeh@umzacir ; ${ }^{3}$ m.usefy@yahoo.com

\section{Abstract:}

Let $\mathcal{B}(\mathcal{H})$ be the algebra of all bounded linear operators on an infinite dimensional Hilbert space $\mathcal{H}$. In this paper, we identify the form of the unital surjective additive map $\varphi: \mathcal{B}(\mathcal{H}) \rightarrow \mathcal{B}(\mathcal{H})$ which preserves the square zero of $\eta$-Lie product of operators for some scalar $\eta$ with $\eta \neq 0,1,-1$.

Keywords: Preserver problem; Square zero operator; $\eta$-Lie product.

MSC (2010): 46J10, 47B48.

\section{Cite this article as (IEEE citation style):}

A. Taghavi, R. Hosseinzadeh, and M. Yousefi, "Maps preserving

the square zero of $\eta$-Lie product of operators", Proyecciones (Antofagasta, On line), vol. 39, no. 3, pp. 591-597, Jun. 2020, doi: 10.22199/issn.0717-6279-2020-03-0036.

Article copyright: (c) 2020 Ali Taghavi, Roja Hosseinzadeh and Masoomeh Yousefi. This is an open access article distributed under the terms of the Creative Commons Licence, which permits unrestricted use and distribution provided the original author and source are credited.

(cc) BY 


\section{Introduction}

Let $\mathcal{A}$ be a Banach algebra, $A, B \in \mathcal{A}$ and $\eta$ be a scalar. The Lie product, $\eta$ Lie product and triple Jordan products are defined as $[A, B]=A B-B A$, $[A, B]_{\eta}=A B+\eta B A$ and $A * B=A B A$, respectively. In last decade, Many mathematician research on the preserving problems. Specially, maps preserving a certain property of products were often considered, see $[1-4]$, [6], [8] and [10 - 12]. We point to some of them close to our purpose.

Authors in [10], considered the maps that strongly preserve the $\eta$-Lie product, that is $\phi(A) \phi(P)+\eta \phi(P) \phi(A)=A P+\eta P A$, for every $A$, some idempotent $P$ and some scalar $\eta$. Author in [12], identified the forms of bijective maps preserving Lie products from a factor von Neumann algebra into another factor von Neumann algebra.

Let $\mathcal{B}(\mathcal{X})$ be the algebra of all bounded linear operators on a Banach space $\mathcal{X}$. In [4], authors characterized the form of unital surjective maps on $\mathcal{B}(\mathcal{X})$ preserving the nonzero idempotency of product of operators in both directions. Also in [11], authors characterized the form of linear surjective maps on $\mathcal{B}(\mathcal{X})$ preserving the nonzero idempotency of either products of operators or triple Jordan products of operators.

We say an operator $A \in \mathcal{B}(\mathcal{X})$ is a square zero operator, when $A^{2}=0$. Let $\mathcal{B}(\mathcal{H})$ be the algebra of all bounded linear operators on an infinite dimensional Hilbert space $\mathcal{H}$. In this paper, we identify the form of surjective additive map $\phi: \mathcal{B}(\mathcal{H}) \rightarrow \mathcal{B}(\mathcal{H})$ such that $\phi(I)=I$ and preserves the square zero of $\eta$-Lie product of operators for some scalar $\eta$ with $\eta \neq 0,1,-1$. The complete form of our main result is as following:

Main Theorem. Let $\mathcal{B}(\mathcal{H})$ be the algebra of all bounded linear operators on an infinite dimensional Hilbert space $\mathcal{H}$. Let $\phi: \mathcal{B}(\mathcal{H}) \rightarrow \mathcal{B}(\mathcal{H})$ be an unital surjective additive map which satisfies

$$
[A, B]_{\eta}^{2}=0 \Leftrightarrow[\phi(A), \phi(B)]_{\eta}^{2}=0,
$$

for every $A, B \in \mathcal{B}(\mathcal{H})$ and for some scalar $\eta$ with $\eta \neq 0,1,-1$. Then there exists either a bounded invertible linear or a conjugate linear operator $T: \mathcal{H} \rightarrow \mathcal{H}$ such that

$$
\phi(A)=T A T^{-1} \quad \text { or } \quad \phi(A)=T A^{*} T^{-1}
$$

for every $A \in \mathcal{B}(\mathcal{H})$. 


\section{Proofs}

First we recall some notations. We denote by $\mathcal{I}(\mathcal{H})$ the set of all idempotent operators in $\mathcal{B}(\mathcal{H})$. For every nonzero $x, y \in \mathcal{H}$, the symbol $x \otimes y$ stands for the rank one linear operator on $\mathcal{H}$ defined by $(x \otimes y) z=<z, y>x$ for any $z \in \mathcal{H}$. Note that every rank one operator in $\mathcal{B}(\mathcal{H})$ can be written in this way.

The rank one operator $x \otimes y$ is idempotent if and only if $\langle x, y\rangle=1$. Let $P, Q \in \mathcal{B}(\mathcal{H})$ be idempotent operators. We say that $P$ and $Q$ are orthogonal if and only if $P Q=Q P=0$.

Proposition 2.1. Let $A \in \mathcal{B}(\mathcal{H}), x, y \in \mathcal{H}$ such that $\langle x, y\rangle=1$ and let $\eta$ be a scalar such that $\eta \neq 0,1,-1$. Then $[A, x \otimes y]_{\eta}^{2}=0$ if and only if only one of the following statements occurs: (i) $A x<A x, y\rangle=-\eta x\left\langle A^{2} x, y\right\rangle$ and $A x=-\eta x<A x, y>$. (ii) $A^{*} y=0$.

Proof. Assume first that $A x<A x, y\rangle=-\eta x<A^{2} x, y>$ and $A x=-\eta x<A x, y>$. Hence

$$
\begin{aligned}
& {[A, x \otimes y]_{\eta}^{2}=\left(A x \otimes y+\eta x \otimes A^{*} y\right)^{2}} \\
& =<A x, y>A x \otimes y+\eta A x \otimes A^{*} y \\
& +\eta^{2}<A x, y>x \otimes A^{*} y+\eta<A^{2} x, y>x \otimes y \\
& =-\eta x<A^{2} x, y>\otimes y-\eta^{2} x<A x, y>\otimes y A \\
& +\eta^{2}<A x, y>x \otimes A^{*} y+\eta<A^{2} x, y>x \otimes y=0 .
\end{aligned}
$$

Now if $A^{*} y=0$, then

$$
\begin{aligned}
& {[A, x \otimes y]_{\eta}^{2}=\left(A x \otimes y+\eta x \otimes A^{*} y\right)^{2}} \\
& =(A x \otimes y)^{2}=<A x, y>A x \otimes y \\
& =<x, A^{*} y>A x \otimes y=0 .
\end{aligned}
$$

Conversely, Assume that $[A, x \otimes y]_{\eta}^{2}=0$. It is clear that

$$
B^{2}=0 \Leftrightarrow B(B x)=0, \quad \forall x \in \mathcal{X} \Leftrightarrow \operatorname{Im} \mathrm{B} \subseteq \operatorname{ker} B .
$$

This together with assumption implies

$$
[A, x \otimes y]_{\eta}^{2}=0 \Leftrightarrow \operatorname{Im}[A, x \otimes y]_{\eta} \subseteq \operatorname{ker}[A, x \otimes y]_{\eta} .
$$


Let $A^{*} y \neq 0$. If $A^{*} y$ and $y$ are linearly independent, then In the following lemmas, assume that $\phi: \mathcal{B}(\mathcal{H}) \rightarrow \mathcal{B}(\mathcal{H})$ is an unital surjective additive map which satisfies

$$
[A, B]_{\eta}^{2}=0 \Leftrightarrow[\phi(A), \phi(B)]_{\eta}^{2}=0,
$$

for every $A, B \in \mathcal{B}(\mathcal{H})$ and for some scalar $\eta$ with $\eta \neq 0,1,-1$.

Lemma 2.2. $\phi$ preserves the square zero operators in both directions.

Proof. Let $A \in \mathcal{B}(\mathcal{H})$. By assumptions we have

$$
\begin{aligned}
A^{2}=0 & \Leftrightarrow(1+\eta)^{2} A^{2}=[A, I]_{\eta}^{2}=0 \\
& \Leftrightarrow[\phi(A), I]_{\eta}^{2}=0 \\
& \Leftrightarrow(1+\eta)^{2} \phi(A)^{2}=0 \\
& \Leftrightarrow \phi(A)^{2}=0
\end{aligned}
$$

The following theorem is a straightforward consequence of Theorem 2.1 in [7].

Theorem 2.3. Let $\mathcal{H}$ be an infinite dimensional Hilbert space and $\phi$ : $\mathcal{B}(\mathcal{H}) \longrightarrow \mathcal{B}(\mathcal{H})$ be a surjective additive map satisfying $\phi(I)=I$. Assume that $\phi$ preserves the square zero operators in both directions. Then $\phi$ is injective and preserves the idempotent operators in both directions.

Lemma 2.4. $\phi$ is injective and preserves the idempotent operators in both directions.

Proof. It is clear by assumptions and Theorem 2.3.

Lemma 2.5. There exists either a bounded invertible linear or a conjugate linear operator $T: \mathcal{H} \rightarrow \mathcal{H}$ such that

$$
\phi(P)=T P T^{-1}
$$

or

$$
\phi(P)=T P^{*} T^{-1}
$$

for every $P \in \mathcal{I}(\mathcal{H})$. 
Proof. Since $\phi$ is additive and by Lemma 2.4 preserves idempotent operators in both directions, then $\phi$ preserves the orthogonality of idempotent operators in both directions. Thus we can obtain the form of $\phi$ on idempotents by Lemma 3.1 in [5].

Remark 2.6. Let $T$ be the same operator defined in Lemma 2.5. It is clear that $\Psi=T^{-1} \phi T: \mathcal{B}(\mathcal{H}) \rightarrow \mathcal{B}(\mathcal{H})$ satisfies the assumptions on $\phi$. Therefore, without loss of generality we can assume that $\phi(P)=P$ or $\phi(P)=P^{*}$ for every $P \in \mathcal{I}(\mathcal{H})$.

Now we are in a position to prove our main result.

Proof of Main Theorem. Let $A \in \mathcal{B}(\mathcal{H})$ such that $\operatorname{ker} A \neq 0$. Let $x \in \operatorname{ker} A$ be nonzero. Hence there exists a nonzero vector $y \in \mathcal{H}$ such that $\langle x, y\rangle=1$. Let the first case of Lemma 2.5 occurs. So by Remark 2.6, $\phi(x \otimes y)=x \otimes y$. By $A x=0$ and Proposition 2.1 we infer that $[A, x \otimes y]_{\eta}^{2}=0$ and by assumption

$$
[\phi(A), \phi(x \otimes y)]_{\eta}^{2}=[\phi(A), x \otimes y]_{\eta}^{2}=0 .
$$

Using again Proposition 2.1 implies

$$
\phi(A) x<\phi(A) x, y>=-\eta x<\phi(A)^{2} x, y>
$$

and

$$
\phi(A) x=-\eta x<\phi(A) x, y>
$$

or $\phi(A)^{*} y=0$. We assert that $\phi(A) x=0$. We assume on the contrary that $\phi(A) x \neq 0$. Let us first assume that (1) and (2) occur.

Thus

$$
-\eta x<\phi(A) x, y>^{2}=-\eta x<\phi(A)^{2} x, y>
$$

and since $\eta \neq 0,<\phi(A) x, y>^{2}=<\phi(A)^{2} x, y>$. It easily follows that $x$, $\phi(A) x$ and $\phi(A)^{2} x$ are linearly dependent, because otherwise, there exists a vector $y$ such that $\langle x, y\rangle=1$ and $\left.\langle\phi(A) x, y\rangle^{2} \neq<\phi(A)^{2} x, y\right\rangle$.

If $x$ and $\phi(A) x$ are linearly dependent, then $\phi(A) x=\alpha x$ for some nonzero scalar $\alpha$. From (2) we obtain $\alpha x\langle x, y\rangle=-\eta \alpha x\langle x, y\rangle$ which implies that $\eta=-1$, that is a contradiction. If $x$ and $\phi(A) x$ are linearly independent, then we conclude that $\phi(A)^{2} x \in \operatorname{span}\{\phi(A) x, x\}$ and so $\phi(A)^{2} x=\alpha \phi(A) x+\beta x$ for some scalars $\alpha, \beta$. It implies that $<\phi(A)^{2} x, y>=\alpha<\phi(A) x, y>+\beta$. According to (1)

$$
\phi(A) x<\phi(A) x, y>=-\eta x(\alpha<\phi(A) x, y>+\beta) .
$$


Since $x$ and $\phi(A) x$ are linearly independent, $\langle\phi(A) x, y\rangle=0$ which by $(2)$ implies, $\langle x, y\rangle=0$, a contradiction.

Now let $\phi(A)^{*} y=0$. Since we assume that $\phi(A) x \neq 0$, there exists a vector $y$ such that $\langle x, y\rangle=1$ and $\langle\phi(A) x, y\rangle \neq 0$. This implies $<x, \phi(A)^{*} y>\neq 0$. It is a contradiction, because $\phi(A)^{*} y=0$. The proof of assertion is completed and so $\operatorname{ker} A \subseteq \operatorname{ker} \phi(A)$, when $\operatorname{ker} A \neq 0$. This implies that if $\operatorname{ker} A \neq 0$, then $\operatorname{ker} \phi(A) \neq 0$ and this with a similar discussion as above yields that $\operatorname{ker} \phi(A) \subseteq \operatorname{ker} A$. Therefore, $\operatorname{ker} \phi(A)=\operatorname{ker} A$ for every operator $A$ such that $\operatorname{ker} A \neq 0$. Moreover, this implies that $\operatorname{ker} A \neq 0$ if and only if $\operatorname{ker} \phi(A) \neq 0$ which yields that $\operatorname{ker} A=0$ if and only if $\operatorname{ker} \phi(A)=0$. Hence $\operatorname{ker} \phi(A)=\operatorname{ker} A$ for every operator $A$ and so $F(\phi(A))=F(A)$ and since $\phi$ is additive, $F(\phi(A)+\phi(B))=F(A+B)$ for every $A, B \in \mathcal{B}(\mathcal{H})$. The form of such $\phi$ has been given in [9], Theorem 3.5. By this theorem, $\phi(A)=U A+R$ such that $U=I-2 \phi(0)$ and $R=\phi(0)$. Since $\phi$ is additive, $\phi(A)=A$. With a similar discussion, we obtain $\phi(A)=A^{*}$, when the second case in Lemma 2.5 occurs. These together with Remark 2.6 complete the proof.

\section{References}

[1] M. A. Chebotar, W.-F. Ke, P.-H. Lee, and N.-C. Wong, "Mappings preserving zero products", Studia mathematica, vol. 155, no. 1, pp. 77-94, 2003, doi: $10.4064 / \mathrm{sm} 155-1-6$

[2] M. Dobovišek, B. Kuzma, G. Lešnjak, C. Li, and T. Petek, "Mappings that preserve pairs of operators with zero triple Jordan product", Linear algebra and its applications, vol. 426, no. 2-3, pp. 255-279, Oct. 2007, doi: 10.1016/j.laa.2007.04.017

[3] G. Dolinar, S. Du, J. Hou, and P. Legiša, "General preservers of invariant subspace lattices", Linear algebra and its applications, vol. 429, no. 1, pp. 100-109, Jul. 2008, doi: 10.1016/j.laa.2008.02.007

[4] L. Fang, G. Ji, and Y. Pang, "Maps preserving the idempotency of products of operators", Linear algebra and its applications, vol. 426, no. 1, pp. 40-52, Oct. 2007, doi: 10.1016/j.laa.2007.03.030

[5] J. Hou and L. Huang, "Maps completely preserving idempotents and maps completely preserving square-zero operators", Israel journal of mathematics, vol. 176, no. 1, pp. 363-380, Mar. 2010, doi: 10.1007/s11856-010-0032-y

[6] L. Molnár, "Non-linear Jordan triple automorphisms of sets of selfadjoint matrices and operators", Studia mathematica, vol. 173, no. 1, pp. 39-48, 2006, doi: 10.4064/sm173-1-3 
[7] P. Šemrl, "Linear mappings that preserve operators annihilated by a polynomial", Journal of operator theory, 36, pp. 45-58, 1996. [On line]. Available: https://bit.ly/2ZCJnB1

[8] A. Taghavi and R. Hosseinzadeh, "Maps preserving the dimension of fixed points of products of operators", Linear and multilinear algebra, vol. 62, no. 10, pp. 1285-1292, Sep. 2013, doi: $10.1080 / 03081087.2013 .823680$

[9] A. Taghavi, R. Hosseinzadeh, and H. Rohi, "Maps preserving the fixed points of sum of operators", Operators and matrices, no. 3, pp. 563-569, 2015, doi: 10.7153/oam-09-34

[10] A. Taghavi, F. Kolivand, and H. Rohi, "A note on strong $\eta$-Lie products preserving maps on some algebras", Mediterranean journal of mathematics, vol. 14, no. 1, 2017, doi: 10.1007/s00009-016-0824-3

[11] M. Wang, L. Fang, and G. Ji, "Linear maps preserving idempotency of products or triple jordan products of operators", Linear algebra and its applications, vol. 429, no. 1, pp. 181-189, Jul. 2008, doi: 10.1016/j.laa.2008.02.013

[12] J.-H. Zhang and F.-J. Zhang, "Nonlinear maps preserving Lie products on factor von Neumann algebras", Linear algebra and its applications, vol. 429, no. 1, pp. 18-30, Jul. 2008, doi: 10.1016/j.laa.2008.01.031 